\title{
Spatial Frequency Selection in Lorentz 4D-Scanning Transmission Electron Microscopy Reconstruction
}

\author{
Binbin Wang ${ }^{1}$, Nuria Bagues ${ }^{2}$, Tao Liu ${ }^{2}$, Jiaqiang Yan $^{3}$, Roland Kawakami ${ }^{2}$ and David McComb ${ }^{1,4}$ \\ ${ }^{1}$ Center for Electron Microscopy and Analysis, The Ohio State University, Columbus, Ohio, United \\ States, ${ }^{2}$ The Ohio State University, Columbus, Ohio, United States, ${ }^{3}$ Oak Ridge National Laboratory, Oak \\ Ridge, Tennessee, United States, ${ }^{4}$ Department of Material Science and Engineering, The Ohio State \\ University, Columbus, Ohio, United States
}

Convergence beam electron diffraction (CBED) has been widely used for structural and electronic investigation of the specimen in scanning transmission electron microscopy (STEM) [1-4]. According to the theory of Ehrenfest [5], the momentum transfer in the diffraction pattern is related to the vertical electric field and Lorentz forces under the probe positions. By measuring the intensity distribution in the diffraction patterns, many recent efforts are given to the phase contrast image reconstruction of the electrostatic/magnetic field from the 4D-STEM dataset with the development of multiple fast electron microscopy detectors [6-9].

For a weak phase object, the contrast transfer function (CTF) of differential phase contrast (DPC) was calculated to depend on the collection angles of the zero disk $[10,11]$ : the central region of the zero disk can only transfer spatial frequencies around convergence angle $(\alpha)$ while the edge region transfers the spatial frequency range from $0-2 \alpha$. Therefore, different spatial frequency signals can be selected in the reconstruction process of 4D-STEM by optimizing convergence angles and scattering angle ranges in the zero disk. For example, in Fig.1, the center of mass (CoM) DPC images of MgO (110) with different spatial frequency selection can be controllably reconstructed from simulated 4D-STEM datasets. For low spatial frequencies, i.e. the probe distribution is far less than the range of the electric/magnetic field, the electric/magnetic field can be estimated from the beam deflection because only a narrow range at the edge of the zero disk is relative to the low spatial frequency [12]. It has been shown that magnetic contrast in DPC images will be improved by using only the reconstruction from the edge of the zero disk [13, 14].

In this work, the spatial frequency selection method was used on Lorentz 4D-STEM dataset acquired using an FEI Titan 60-300 TEM fitted with an EMPAD detector using (a) bulk FeGe, (b) confined bulk FeGe and (c) plan-view FeGe polycrystalline thin film $(35 \mathrm{~nm})$ that grown on Si substrate. Examples of reconstructed magnitude images of the skyrmion magnetic field from full zero disk and edge region with bulk and confined bulk FeGe samples are shown in Fig.2. For the bulk FeGe, two images (Fig.2a\&2b) show comparable skyrmion lattice with slightly enhanced contrast for high frequency signal in the one reconstructed from the edge region (black arrows in Fig.2e). However, the frequency filtered image of the confined FeGe (Fig.2f) show improved magnetic contrast than the full disk one in Fig.2g because the midfrequency diffraction contrast background (Fig.2h) is reduced by cutting out the inner zero disk region. The same procedure has also proved to be useful for plan-view FeGe polycrystalline thin film which is prepared by wedge-mechanical polishing and is further complexed by diffraction, defects and grain contrasts. By properly select the convergence and scattering angles, we had successfully disentangled the helical states in regions supported by Si substrate, which is inconsistent with experiments that interpret the deficient of helical states in FeGe films on Si substrate as they have easy-plane anisotropy that may prevent skyrmion formation $[9,15,19]$. 


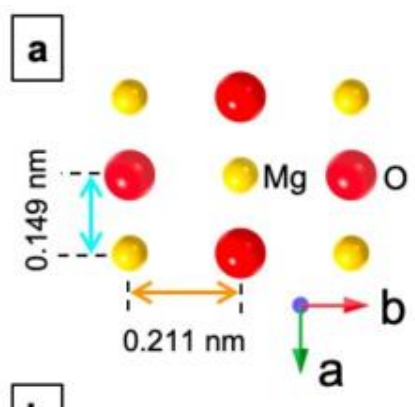

b

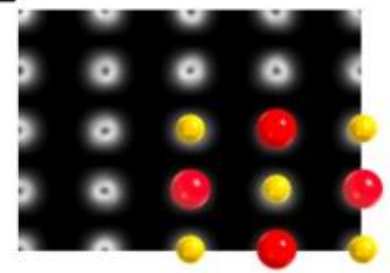

Projected e-field (Isolated single atom model)
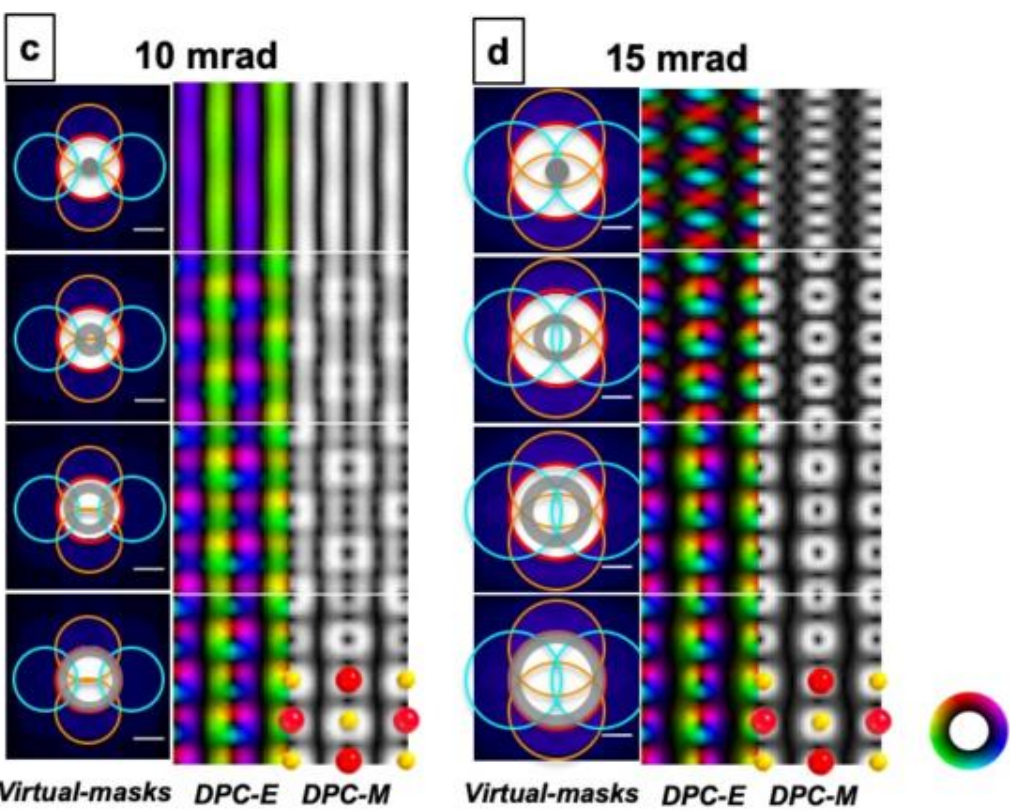

Figure 1. (a) the crystal model of $\mathrm{MgO}$ (110). (b) the projected electrostatic field of $\mathrm{MgO}$ (110) which is calculated from the projected potential in our simulation model with isolated single atom model. The middle and right column in (c) and (d) are the reconstructed electrostatic polar figure and its magnitude with different virtual masks at different scattering range from 4D-STEM dataset with convergence angle to be $10 \mathrm{mrad}$ in (c) and $15 \mathrm{mrad}$ in (d) (gray annular in the left diagram while the red circle indicates the zero disk). The thickness of the crystal model in this simulation was $1 \mathrm{~nm} . \mu$ STEM [16], py4DSTEM [17], and pixSTEM [18] was involved in our simulation. 


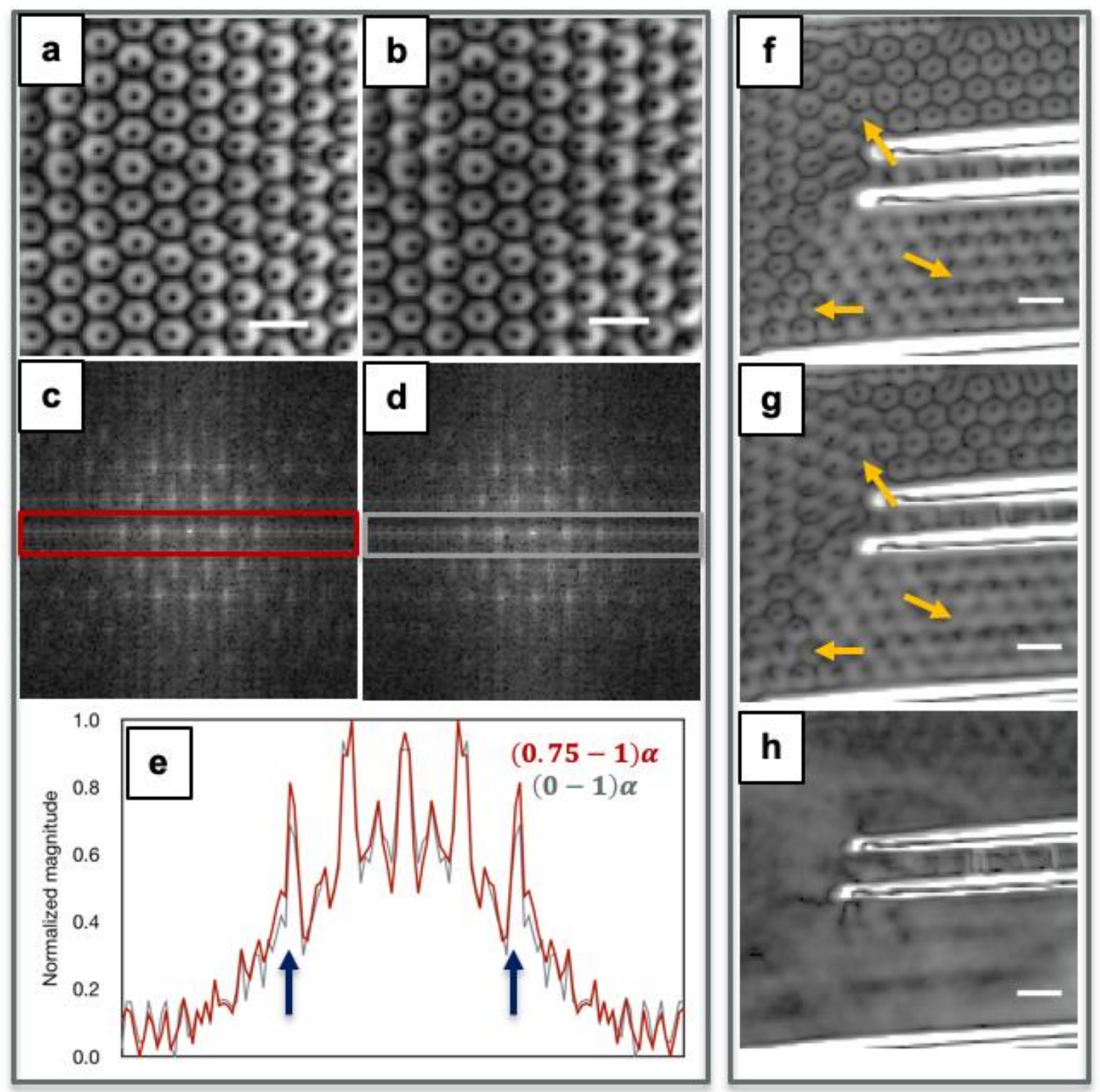

Figure 2. Magnitude images of magnetic field of skyrmion lattice reconstructed from bulk FeGe sample with edge region (0.75-1) $\alpha$ in (a) and full zero disk (0-1) $\alpha$ in (b). The fast Fourier translation was performed on (a) and (b), and its amplitude patterns are shown in (c) and (d) separately. The magnitude profile along the stripe indicated in (c) and (d) are normalized in (e) to show the difference in reconstructed images. Magnitude images of magnetic field of skyrmion in the confined bulk FeGe sample were constructed with scattering angle range (0.75-1) $\alpha$ in (f), (0-1) $\alpha$ in $(\mathrm{g})$ and (0-0.75) $\alpha$ in $(\mathrm{h})$. All images are acquired at $260 \mathrm{~K}$ with $50( \pm 10) \mathrm{mT}$. Background correction and image contrast was modified in same for each image. The scale bar is $150 \mathrm{~nm}$.

\section{References}

[1] C Ophus, Microsc Microanal 25 (2019), p. 563.

[2] J Zuo et al., Nature 401 (1999), p. 49.

[3] JM LeBeau et al., Ultramicroscopy 110 (2010), p. 118.

[4] B Wang et al., Physical Review Research 1 (2019), p. 032037.

[5] B Lippmann, Phys Rev Lett 15 (1965), p. 11. 
[6] Y Jiang et al., Nature 559 (2018), p. 343.

[7] W Gao et al., Nature 575 (2019), p. 480.

[8] Z Chen et al., Microsc Microanal 25 (2019), p. 32.

[9] KX Nguyen et al., arXiv preprint arXiv:200106900, (2020), p.

[10] H Yang, TJ Pennycook and PD Nellist, Ultramicroscopy 151 (2015), p. 232.

[11] M Lohr et al., Ultramicroscopy 117 (2012), p. 7.

[12] K Müller-Caspary et al., Ultramicroscopy 178 (2017), p. 62.

[13] S Majert and H Kohl, Ultramicroscopy 148 (2015), p. 81.

[14] M Krajnak et al., Ultramicroscopy 165 (2016), p. 42.

[15] M Wilson et al., Phys Rev B 89 (2014), p. 094411.

[16] LJ Allen and S Findlay, Ultramicroscopy 151 (2015), p. 11.

[17] BH Savitzky et al., Microsc Microanal 25 (2019), p. 124.

[18] pixStem, https://pixstem.org (accessed February 21, 2019).

[19] The authors acknowledge funding from Defense Advanced Research Projects Agency (DARPA) under Grant No. D18AP00008. 